\title{
Application of microsatellite markers in identifying genetic relationship among different swine populations in Sri Lanka
}

\author{
E. Subalini', G.L.L.P. Silva ${ }^{2}$ and C.M.B. Dematawewa ${ }^{2}$ \\ ${ }^{\prime}$ Department of Animal Science, Faculty of Agriculture, Eastern University, Chenkalady, Sri Lanka \\ ${ }^{2}$ Department of Animal Science, Faculty of Agriculture, University of Peradeniya, Peradeniya, Sri Lanka
}

\begin{abstract}
Five different swine populations in Sri Lanka inchuding Village pigs, wild boar and exotic breeds such as Large White, Landrace and Duroc were selected for this genetic study. Fifteen microsatellite markers recommended by the FAO (Food and Agriculture Organization) and ISAG (International Society of Animal Genetics) were employed to analyze genetic distance among different swine populations. A total of 120 samples of pigs were collected from various locations in Sri Lanka where village pigs and wild boar could be found. The MICROSAT and PHYLIP computer packages were used to estimate the allelic frequency and construct the phylogenetic tree. The phylogenetic tree was constructed using Neighbor-joining approach with 1000 bootstrap values. The results of the study revealed that 138 alleles were found at 15 loci screened across five swine populations. Among them 11 were polymorphic and rest were monomorphic. The polymorphic alleles ranged between 2-4 alleles per locus. The genetic distance between village pig and wild boar (0.091) was closer than between Large White (0.261), Landrace (0.241) and Duroc (0.268). According to the dendogram the village pig and wild boar populations clearly diverged from exotic breeds. In the phylogenetic tree, high distances observed were separating exotic pig populations from the other two populations of village pigs and wild boar. Further, the village pigs and wild boar clustered separately. Based on these findings it is proposed that the village pigs and wild boars may be recognized as distinct population for breeding or conservation purpose.
\end{abstract}

ABSTRACT

Keywords: Microsatellite, swine, village pigs, wild boar

\section{Introduction}

Several DNA-based technologies to type the polymorphic loci have been developed in the last decade. These techniques include the Restriction Fragment Length Polymorphisms (RFLP), Variable Number of Tandem Repeats (VNTR), Single Strand Conformational Polymorphism (SSCP), Denaturing Gradient Gel Electrophoresis (DGGE), Random Amplified Polymorphic DNA (RAPD) and also the methods which make use of the polymorphism of the Short Tandem Repeats (STR) called the microsatellites. Microsatellites are the short segments of the DNA in which a specific motif is tandomly repeated.

The microsatellite polymorphisms are now used for a wide range of applications in genetics including the genetic distancing studies between the breeds or populations or individuals, linkage mapping of the disease genes and the quantitative traits, paternity testing and individual identification for selective breeding (Bruford and Wayne, 1993; Goldstcin and Schlotterer, 1999). As compared to most other types of DNA sequences, the microsatellites are the highly polymorphic which makes them attractive as genetic markers (Goldstein and Shlotterer, 1999). The allelic fiequency data of microsatellite obtained after the polymerase chain reaction-based genome scoring can be utilized for studying the evclutionary relationships of the closely related breeds or populations of a species or closely related species (Laval et al., 2001). The more and more reports are now appearing in the farm animals including pigs describing genetic characterization with microsatellite markers (Fredholm et al., 1993; Li et al., 2001 ; Subalini et al., 2009 and Van Zeveran et al., 1995). 
The indigenous pigs in Sri Lanka are popular for their quality and tasty meat. However, in Sri Lanka there is no planned breeding program for these pigs and as a result the indigenous pig population is decreasing gradually. This is mainly due to lack of attention in their improvement and the swine industry is mainly oriented towards the exotic germplasm leaving behind the most valuable genetic resource exist within the indigenous pigs.

The present breeds of idomesticated pigs (Sus domesticus) are originated from two wild ancestors such as' Sus scrofa (wild boar) and Sus vittatus (wild pig). Their genetic diversity has provided the material for the very successful pig breeding and improvement programs of the developed world in $19^{\text {th }}$ and $20^{\text {th }}$ century. These wild ancestors represent a unique resource respond to the present and future needs of swine production both in developed and developing countries.

The molecular investigation is important to identify the possible gene flow between wild and village pig populations and will facilitate its sustainable management or utilization. In Sri Lanka some researchers have evaluated the village and wild type pigs at phenotypic level on several occasions. However, no reports are available to identify the genetic relatedness among swine population at molecular level.

In this context, this study was formulated to identify genetic variation among pig populations and find the degree of genetic relatedness among village pigs, wild boar and domesticated populations. The information generated will be used to compare the level of diversity and uniqueness of the local population using the available databases established by the FAO (Food \& Agriculture Organization). In the long run the results of the study will contribute to establish genetic improvement program for native pigs to facilitalc their contribution in rural economy and nutrition.

The genetic diversity studies are currently carried out by using molecular tecliniques that include marker based genome screening. Microsiltllites are currently the markers of choice for the dutcition of genetic relatedness in livestock species due to their abundance, ubiquitous distribution, polymorphic nature, and suitability for amplification by polymerase chain reaction (PCR). Microsatellite is a PCR (Polymerase Chain Reaction) based marker and relatively cost effective in identifying the diversity in populations. Therefore, present investigation was done by using . microsatellite markers.

\section{Materials and Methods}

The Laboratory studies were carried out at Agricultural Biotechnology Center, Faculty of Agriculture, University of Peradeniya, Sri Lanka. A total of hundred and twenty unrelated village pigs, wild boars and exotic pigs were sampled from several farmholdings from selected areas. Village pigs were sampled from Kalutara, Kurunegala, Puttalam and Chilaw while wild boar sampled from Kandy, Batticaloa, Trincomalee, Polonnaruwa and Anuradhapura. Exotic pig samples were collected from Welisara Swine Breeding Station, Chutyduwa Private Swine Farm and Udaperadeniya Farm, University of Peradeniya, Kandy. The areas for sample collection were selected where the particular swine population is high. Extraction of genomic DNA, quantification of DNA, Polymerase Chain Reaction, Polyacrylamide Gel Electrophoresis and silver staining were done during laboratory investigation.

\section{Blood sample collection for DNA extraction}

Blood samples were collected under sterile conditions without cross contamination using fresh needle (18 guage $\times 1.5$ inches) for each animal. Five milliliters of blood from ear vein was collected from village pigs and exotic pigs. Blood from wild boar was collected during slaughtering and also from vein on hind limb after sedation of animal depending on the situation. Blood sample was collected into vacutainer containing anticoagulant $(200 \mathrm{ml}$ of $0.129 \mathrm{mM}$ Sodium citrate per $500 \mathrm{ml}$ of blood) and transported on ice immediately to the laboratory and stored in $4^{\circ} \mathrm{C}$ until the analysis is carried out.

\section{DNA extraction and quantification}

The genomic DNA was extracted from the collected blood samples using salting out protocol. Extracted DNA was stored at $-20^{\circ} \mathrm{C}$ until used. Quantification of DNA was done using a spectrophotometer (UV visible spectrometer, 
model-cintra $10 \mathrm{e})$. Reading was taken at 2 wave lengths; $260 \mathrm{~nm}$ and $280 \mathrm{~nm}$. In addition to the quantification using the spectrophotometer samples were subjected to agarose gel ectrophoresis to confirm the presence of DNA. All the DNA samples were run in $0.8 \%$ agarose gel with $1 \mathrm{~kb}$ ladder. The gel was allowed to run at $60 \mathrm{~V}$ until bromophenol blue has migrated to an appropriate distance (about 1 hour). The gel was visualized using High Performance Ultra-Violet Transilluminator and photographed.

\section{Polymerase Chain Reaction with microsatellites}

Polymerase Chain Reaction was carried out using thermocycler (TECHINE, model- TC 4I2). Amplification of DNA fragments was carried out using 15 swine microsatellite primers namely SW911, SWR198, SW252, SW256, SW310, SW 1067, SW 1089, S0090, S0218, S0226, SWI22, SW2008, S0228, SW355 and SW335. The primers were selected based on ISAG/FAO (International Society for Animal Genetics/ Food and Agriculture Organization) recommendation and attention was paid to select primers covering the whole genome as far as possible. Polymerase Chain Reaction conditions were optimized for each primer. The cocktail mixture was prepared using distilled water, $10 X$ Buffer (ABgene, UK), $\mathrm{MgCl}_{2}$ (ABgene, UK), dNTP (2.5mM, ABgene, UK), Forward primer $(20 \mu \mathrm{M})$, Reverse Primer $(20 \mu \mathrm{M})$, Tag polymerase

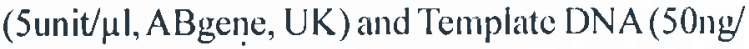
$\mu \mathrm{l})$.

\section{Amplification conditions for primers}

Amplification was carried out using thermocycler (TEÇHINE, model- TC 4I2) according to the manufacture's instruction (TIBMOLBIOL, Germany). The initial denaturation and extension temperature were same for all primers but the annealing temperature were different in different primers. The amplification conditions for each primer were, initial denaturation $94^{\prime \prime} \mathrm{C} / 5$ minutes, first denaturation $94^{\circ} \mathrm{C} / 45$ seconds, initial extension $72^{\circ} \mathrm{C} / 1$ minute, final extension $72^{\circ} \mathrm{C} / 10$ minutes and final holding temperature $4^{\prime \prime} \mathrm{C}$ with 30 cycles and varying range of annealing temperature for each primer. Polymerase chain reaction products were then subjected to agarose gel electrophoresis (2\%) at $40 \mathrm{~V}$ for 2 to $21 / 2$ hours to confirm its presence. The Polyacrylamide Agarose Gel Electrophoresis was carried out subsequently for genotyping of samples and allelic quantilication.

\section{Polyacrylamide Gel Electrophoresis (PAGE) and Silver staining}

Two microliter of loading buffer solution was mixed with $4 \mu$ of amplified PCR product in a new set of PCR tubes. Then the samples were denatured at $94^{\circ} \mathrm{C}$ for 5 minutes and tubes were dipped into ice immediately for snap cooling. Sample was run at $1200 \mathrm{~V}$ until the bromophenol blue and xylene cynole FF migrated to an appropriate distance through the gel (around 5 to 6 hours). Silver staining was performed at last to get visualized the DNA bands for scoring.

\section{Molccular analysis}

MICROSAT (Version 1.5d) and PHYLIP computer packages were used to estimate allelic frequency and to construct phylogenetic tree. The phylogenetic tree was constructed with 1000 bootstrap values.

\section{Results and Discussion}

\section{Allele distribution}

The application of molecular methods in evaluating the genetic diversity makes it possible to estimate the genetic variability between and within commercial and indigenous strains, measure the genetic distances and similarities between different strains, breeds or lines and assess the genetic heterozygosity. For this analysis allele frequencies for 15 microsatellites were used in five populations consisting of wild boar, village pig, and 3 exotic swine populations (Landrace, Large White and Duroc). Allele frequencies for each locus were calculated using diploid chromosome number $(2 n=50)$ for each swine type. In total, 138 alleles were found at the 15 loci screened across live populations. Among the 15 microsatellite loci 11 were polymorphic and the rest (SW91I, SW2008, SW 1089) and SW335) were monomorphic. The polymorphic alleles ranged between 2 alleles to 4 alleles per locus. 


\section{Genetic distances between populations}

Genetic distances between populations were calculated using Nei estimator and the results are shown in Table 1. Based on allelic frequency of 15 microsatellites loci, the genetic distances $\left(D_{N}\right)$ between village pigs and wild boar was found to be 0.091 . The genetic distance between village pigs and, Large White, Landrace and Duroc were found to be $0.261,0.241$ and $Q .268$, respectively
(Table 1), showing that the village pigs formed a distinct population. This observation confirms the fact that the evolutionary path of village pig is completely separated from the evolutionary path of exotic pigs. The genetic distances of wild boar and village pigs was comparatively closer than between village pigs and cxotic pigs indicated that these both populations have been originated from common source of ancestor or there has been gene flow between these populations.

Table 1: Genetic distances estimated among five swine populations

\begin{tabular}{ccclcc}
\hline Population & 1 & 2 & 3 & 4 & 5 \\
\hline 1 & - & & & & \\
2 & 0.091 & - & - & & \\
3 & 0.262 & 0.261 & 0.053 & - & - \\
4 & 0.244 & 0.241 & 0.040 & 0.040 & 5-Duroc \\
5 & 0.284 & 0.268 & 3-Large White & 4-Landrace &
\end{tabular}

As Subalini ei al. 2010 stated on a report of phenotypic study of native pigs that in some areas village pigs kept under extensive management system moved freely along the forest areas which facilitate free mating between these swine populations. This finding is in agreement with the results of Chinese study by Zhao et $a /(1990)$ and Zang el al (2003) stated that the gene flow from wild animals to native populations can frequently be observed in swine.

The village pigs closely resemble the Sri Lankan wild boars and must have evolved as a result of gradual domestication of wild boars. The presence of horizontal stripes in newborn piglings of village pigs which gradually disappear with age is further proof of the native pigs' wild ancestry. The tendency to use all four toes while standing and walking also links the native type to wild pigs, as it is a characteristic of wild boar to use all four toes to get more footing in their natural habitat (Fisher and Devendra, 1963). The exotic pigs, in contrast use only the two front toes.

Further, the phenotypic dissection and comparison between these native pig types revealed that the village pigs in some locations showed similar morphology of wild boar such as village pigs had long straight face, upwardly erected ears, angular body shape and long hairs densely found alongthe spine. The dendogram obtained from these distances with PHYLIP method is displayed in Figure I.

\section{Dendogram}

The dendogram (Figure 1) showing the genetic distance $\left(D_{A}\right.$ developed using the neighbor joining approach (with 1000 bootstrap) indicates that there is a clear separation of village pigs and wild boars from the other three exotic groups. Duroc and Large white are more closely related with each other than with Landrace. However, despite the clear separation of native Sri Lankan pigs from the exotic breeds, the genetic distance between the two native groups; village pigs and wild boars is substantial. It shows that the village pigs and wild boar populations do not have a very close genetic relationship at present though they might have originated from a common source.

On the basis of these results, it is evident that both village pigs and wild boar need equal attention in conservation attempts. However, it is advisable to expand the comparison with other native pig types of the region before coming to a conclusion on conservation programme. 


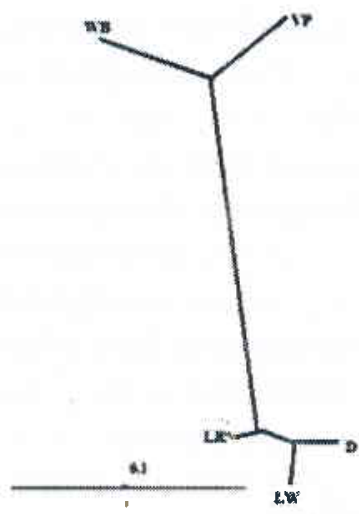

Figure 1.The unrooted neighbor joining tree relating the allelic distribution of fifteen microsatellite markers in five different swine populations. (WB- Wild boar, VPVillage pigs, LW-Large White, LR-Landrace, D-Duroc)

\section{Conclusions}

The molecular findings revealed that the village pigs and wild boars are two distinct populations. However, both populations might originate from common source of origin. This observation could be very well confirmed by the breeding practice of village pigs and wild boars in the country.

\section{References}

Bruford, M.W. and R.K. Wayne (1993).

Microsatellites and their application to population genetic studiesCurrent opinion : in Genetics and Development, 3: 939-943.

Fischer, H. and C. Devendra (1963). Origin and performance of local swine in Malaya. Paper presented to the FAO Meeting on pig diseases and production. Singapore. Working paper-15.

Fredholm, M., A.K. Wintero., K. Christense., B. Christesen., P.B. Nielson., W. Davies and A. Archibald (1993)Characterization of 24 porcine microsatellites: genotyping of unrelated animals from four breeds and linkage studies. Mammalian Genome 4: 187-192.

Goldstein, D. B. and C. Schlotterer (1999). Microsatellites: evolution and applica tion's. Oxford University Press, New York.
Nei, M. (1980). Estimation of average heluryy gosity and genetic distance from a small number of individuals. Genetics 89: 583590.

Laval, G., N. Iannuccelli., C. Legault., M.A.M. Groenen and E. Giuffra (2000). Genetic diversity of eleven European pig breeds. Genetic Selection and Evolution 32: 187-203.

Li, K., Y. Chen., C. Moran., B. Fan., S. Zhao and $Z$. Peng (2001). Analysis of diversity and genetic relationships between four Chinese indigenous pig breeds and one Australian commercial breed. Animal Genetics 31:322-325.

Subalini, E., G.L.L.P. Silva and C.M.B.

Dematawewa (2010). Phenotypic varia tions between native pig types in Sri Lan ka. Journal of Tropical Agriculture Research, Postgraduate Institute of Agriculture, University of Peradeniya, Sri Lanka. 22 (3): 75-95

Subalini, E., G.L.L.P. Silva and C.M.B. Demata wewa (2010). Phenotypi Characterization and Production Performances of Village pigs in Sri Lanka. Journal of Tropical Agriculture Research, Postgraduate Institute of Agriculture, University of Peradeniya, Sri Lanka. 21 (2): 89-94

Van Zeveran, A., L. Peelman., A. Van de Weghe and Y. Bouguet (1995). A genetic study of four Belgian pig populations by means of seven microsatellite loci. Journal of Animal Breeding and Genetics 112: 191-204.

Zhang, G.X., Z.G. Wang., F.Z. Sun., W.S. Chen., G.Y. Yang, S.J. Guo., Y.J. Li., X.L. Zhao Y. Zhang., J. Sun., B. Fan., S.L. Yang and K. Li (2003). Genetic diversity of micro satellite loci in fifty six Chinese native pig breeds. Yi Chuan Xue Bao. 30 (3): 225-233.

Zhao, Z.L. (1990). The characteristics of Chinese pig breeds. Symposium sur le Porc Chinois (M.Molenat, C. Legault eds), INRA Jouyen-Josas. 56-65. 